\title{
Dynamical Invariant Applied on General Time-Dependent Three Coupled Nano-Optomechanical Oscillators
}

\author{
Sara Hassoul, ${ }^{1}$ Salah Menouar, ${ }^{1}$ Hamid Benseridi, ${ }^{2}$ and Jeong Ryeol Choi $\mathbb{D}^{3}$ \\ ${ }^{1}$ Laboratory of Optoelectronics and Compounds (LOC), Department of Physics, Faculty of Science, University of Ferhat Abbas Setif 1, \\ Setif 19000, Algeria \\ ${ }^{2}$ Laboratory of Applied Mathematics (LaMA), Department of Mathematics, Faculty of Science, University of Ferhat Abbas Setif 1, \\ Setif 19000, Algeria \\ ${ }^{3}$ Department of Nanoengineering, Kyonggi University, Yeongtong-gu, Suwon, Gyeonggi-do 16227, Republic of Korea
}

Correspondence should be addressed to Jeong Ryeol Choi; choiardor@hanmail.net

Received 11 April 2021; Revised 4 June 2021; Accepted 26 June 2021; Published 26 July 2021

Academic Editor: Shweta Dabhi

Copyright (C) 2021 Sara Hassoul et al. This is an open access article distributed under the Creative Commons Attribution License, which permits unrestricted use, distribution, and reproduction in any medium, provided the original work is properly cited.

\begin{abstract}
A quadratic invariant operator for general time-dependent three coupled nano-optomechanical oscillators is investigated. We show that the invariant operator that we have established satisfies the Liouville-von Neumann equation and coincides with its classical counterpart. To diagonalize the invariant, we carry out a unitary transformation of it at first. From such a transformation, the quantal invariant operator reduces to an equal, but a simple one which corresponds to three coupled oscillators with timedependent frequencies and unit masses. Finally, we diagonalize the matrix representation of the transformed invariant by using a unitary matrix. The diagonalized invariant is just the same as the Hamiltonian of three simple oscillators. Thanks to such a diagonalization, we can analyze various dynamical properties of the nano-optomechanical system. Quantum characteristics of the system are investigated as an example, by utilizing the diagonalized invariant. We derive not only the eigenfunctions of the invariant operator, but also the wave functions in the Fock state.
\end{abstract}

\section{Introduction}

Arrays of coupled oscillators are ubiquitous in nature, and their oscillatory motions are usually applied in technological sciences [1-5]. Thanks to this, there has been an increasing interest in the dynamical and statistical behavior of a set of coupled oscillators so far. A wide class of physical phenomena of interacting systems is explainable from the model of coupled harmonic oscillators. It includes nano-optomechanical resonances [6, 7], electromagnetically induced transparency [8], stimulated Raman effects [9], Josephson phenomena [10], one-half spin dynamics [11], and trapping of different particles [1]. Among various issues related to coupled harmonic oscillators, the entanglement and mixedness are the most remarkable characters which give a theoretical basis for quantum technologies, such as quantum computing and quantum cryptography [12-14]. Hence, the entanglement in three coupled harmonic oscillators should be analyzed from the fundamental quantum mechanical point of view.
If we consider the above-mentioned importance of coupled oscillatory systems, the investigation of their dynamical properties are highly required. Because the motion of coupled oscillators is complex, oscillator dynamics requires rigorous description especially when the parameters vary over time and/or the number of coupling is more than two [3]. One of the potential tools for studying oscillator dynamics in the time-dependent regime is the dynamical invariant operator $[15,16]$. The invariant operator theory for timedependent harmonic oscillators is based on the construction of adiabatic invariants which were firstly introduced by Lewis and Riesenfeld $[15,16]$ in describing their quantum features. The analysis of many oscillatory systems was fulfilled by introducing an invariant [13-25]. The dynamical invariant is also useful when we examine the entanglement for timedependent coupled oscillators based on the Schrödinger equation [14].

The aim of this work is to study dynamical invariant of general time-dependent three coupled nano-optomechanical 
oscillators $[6,7,26-28]$. This research is motivated by the usefulness of the dynamical invariant on elucidating classical and quantum mechanical properties of complicated mechanical systems. Considering the powerfulness of the invariant in analyzing mechanical systems, we will formulate a mathematical invariant for general time-dependent three coupled nanooptomechanical oscillators in this work. The obtained invariant will be applied to the analysis of the dynamics of the optomechanical systems. In particular, we will focus on the use of the invariant in unfolding exact quantum theory of the system.

If a time-dependent system is relatively simple, the invariant operator can be directly applied to analyzing the quantum dynamics of the system. However, due to the complexity of the three coupled nano-optomechanical oscillatory systems that we consider, we will diagonalize the invariant via the unitary transformation technique. The diagonalization of the invariant may greatly simplify the analysis of the dynamics of the system. In order to show this, the complete quantum wave functions of the system will be derived using the eigenstates of the invariant operator as an example.

Our work is organized as follows. Our main study starts from Section 2. In Subsection 2.1, we will derive the classical invariant quantity as a preliminary step in our development, starting from the classical equations of motion for the coupled nano-optomechanical oscillators. We will find the quantum invariant operator together with the ladder operators and will simplify it by means of the unitary transformation approach in Subsection 2.2. Matrix representation of this invariant operator will be diognalized in Subsection 2.3 by using the technique of diagonalization in $3 \mathrm{D}$. The eigenfunctions of the invariant operator and wave functions of the system will be evaluated in Section 3. Because the wave functions of the transformed (or diagonalized) system is well known, we can easily find the wave functions of the original system through an inverse transformation. Finally, concluding remarks are given in the last section.

\section{Results and Discussion}

2.1. Construction of the Classical Invariant. We consider three coupled nano-optomechanical oscillators with different masses $m_{i}(t)(i=1,2,3)$ and frequencies $\omega_{i}(t)$, respectively; the convention for $i$ (including $j$ and $k$ ) given here will be used throughout the paper. Such a system is described by the Hamiltonian [29].

$$
\begin{aligned}
H(t)= & \frac{1}{2} \sum_{i=1}^{3}\left[\frac{P_{i}^{2}}{m_{i}(t)}+m_{i}(t) \omega_{i}^{2}(t) X_{i}^{2}\right] \\
& +\frac{1}{2}\left[k_{12}(t) X_{1} X_{2}+k_{13}(t) X_{1} X_{3}+k_{23}(t) X_{2} X_{3}\right],
\end{aligned}
$$

where $X_{i}$ and $P_{i}$ are the canonical coordinates and momenta, and $k_{12}(t), k_{13}(t)$, and $k_{23}(t)$ are coupling parameters. We will derive the classical invariant for this system in this section. To this end, let us first start from the Hamilton's equations of the form

$$
\dot{X}_{i}=\frac{\partial H}{\partial P_{i}}, \quad \dot{P}_{i}=\frac{-\partial H}{\partial X_{i}} .
$$

From minor evaluations with these, we easily see that the classical equations of motion are given by

$$
\begin{aligned}
& \ddot{X}_{1}+\left(\frac{\dot{m}_{1}}{m_{1}}\right) \dot{X}_{1}+\omega_{1}^{2} X_{1}+\left(\frac{k_{12}}{2 m_{1}}\right) X_{2}+\left(\frac{k_{13}}{2 m_{1}}\right) X_{3}=0, \\
& \ddot{X}_{2}+\left(\frac{\dot{m}_{2}}{m_{2}}\right) \dot{X}_{2}+\omega_{2}^{2} X_{2}+\left(\frac{k_{12}}{2 m_{2}}\right) X_{1}+\left(\frac{k_{23}}{2 m_{2}}\right) X_{3}=0, \\
& \ddot{X}_{3}+\left(\frac{\dot{m}_{3}}{m_{3}}\right) \dot{X}_{3}+\omega_{3}^{2} X_{3}+\left(\frac{k_{13}}{2 m_{3}}\right) X_{1}+\left(\frac{k_{23}}{2 m_{3}}\right) X_{2}=0 .
\end{aligned}
$$

There exist in principle many dynamical invariants for any given Hamiltonian $H$. We consider a quadratic invariant indexed by one or more parameters. Let us assume that its formula is given in the form

$$
\begin{aligned}
O(t)= & \frac{1}{2} \sum_{i=1}^{3}\left[A_{i}(t) P_{i}^{2}+B_{i}(t) X_{i} P_{i}+C_{i}(t) X_{i}^{2}\right] \\
& +\frac{1}{2}\left[D_{12}(t) X_{1} X_{2}+D_{13}(t) X_{1} X_{3}+D_{23}(t) X_{2} X_{3}\right]
\end{aligned}
$$

where the time-dependent parameters $A_{i}(t), B_{i}(t), C_{i}(t)$, $D_{12}(t), D_{13}(t)$, and $D_{23}(t)$ are real and differentiable functions, which will be explicitly evaluated later on.

The dynamical invariant $O(t)$ in classical mechanics satisfy the equation

$$
\frac{d O}{d t}=\frac{\partial O}{\partial t}+\sum_{i=1}^{3}\left\{\frac{\partial O}{\partial X_{i}} \frac{\partial H}{\partial P_{i}}-\frac{\partial O}{\partial P_{i}} \frac{\partial H}{\partial X_{i}}\right\}=0 .
$$

To derive the formulae of time-dependent parameters in Equation (6), we carry out some basic evaluations after inserting Equations (1) and (6) into Equation (7). Then, this gives

$$
\begin{aligned}
\dot{A}_{i}(t) & =\frac{-2 B_{i}(t)}{m_{i}(t)}, \\
\dot{B}_{i}(t) & =m_{i}(t) \omega_{i}^{2}(t) A_{i}(t)-\frac{C_{i}(t)}{m_{i}(t)}, \\
\dot{C}_{i}(t) & =2 m_{i}(t) \omega_{i}^{2}(t) B_{i}(t), \\
\dot{D}_{12}(t) & =k_{12}(t)\left[B_{1}(t)+B_{2}(t)\right], \\
\dot{D}_{13}(t) & =k_{13}(t)\left[B_{1}(t)+B_{3}(t)\right], \\
\dot{D}_{23}(t) & =k_{23}(t)\left[B_{2}(t)+B_{3}(t)\right] .
\end{aligned}
$$

Possible solutions for these differential equations are obtained in the following forms 


$$
\begin{aligned}
A_{i}(t) & =\frac{1}{m_{i}(t)}, \\
B_{i}(t) & =\frac{\dot{m}_{i}(t)}{2 m_{i}(t)}, \\
C_{i}(t) & =\int_{0}^{t} \omega_{i}^{2}(t) \dot{m}_{i}(t) d t, \\
D_{12}(t) & =\int_{0}^{t} k_{12}(t)\left(\frac{\dot{m}_{1}(t)}{2 m_{1}(t)}+\frac{\dot{m}_{2}(t)}{2 m_{2}(t)}\right) d t, \\
D_{13}(t) & =\int_{0}^{t} k_{13}(t)\left(\frac{\dot{m}_{1}(t)}{2 m_{1}(t)}+\frac{\dot{m}_{3}(t)}{2 m_{3}(t)}\right) d t, \\
D_{23}(t) & =\int_{0}^{t} k_{23}(t)\left(\frac{\dot{m}_{2}(t)}{2 m_{2}(t)}+\frac{\dot{m}_{3}(t)}{2 m_{3}(t)}\right) d t .
\end{aligned}
$$

In terms of the above solutions of the coupled equations, we can describe the classical invariant $O(t)$ in the form

$$
\begin{aligned}
O(t)= & \frac{1}{2} \sum_{i=1}^{3}\left[\frac{P_{i}^{2}}{m_{i}(t)}+\frac{\dot{m}_{i}(t)}{m_{i}(t)}\left(X_{i} P_{i}\right)+\left(\int_{0}^{t} \omega_{i}^{2}(t) \dot{m}_{i}(t) d t\right) X_{i}^{2}\right] \\
& +\frac{1}{2}\left(\int_{0}^{t} k_{12}(t)\left(\frac{\dot{m}_{1}(t)}{m_{1}(t)}+\frac{\dot{m}_{2}(t)}{m_{2}(t)}\right) d t\right) X_{1} X_{2} \\
& +\frac{1}{2}\left(\int_{0}^{t} k_{13}(t)\left(\frac{\dot{m}_{1}(t)}{m_{1}(t)}+\frac{\dot{m}_{3}(t)}{m_{3}(t)}\right) d t\right) X_{1} X_{3} \\
& +\frac{1}{2}\left(\int_{0}^{t} k_{23}(t)\left(\frac{\dot{m}_{2}(t)}{m_{2}(t)}+\frac{\dot{m}_{3}(t)}{m_{3}(t)}\right) d t\right) X_{2} X_{3} .
\end{aligned}
$$

It is obvious that this invariant is not unique; there may exist many invariants for the given Hamiltonian, because Equations (14)-(19) are not uniquely determined in general.

2.2. Quantum Invariant Operator. The relation between the pair of classical canonical variables, $\left(X_{i}, P_{j}\right)$, and the corresponding quantum variables, $\left(\widehat{X}_{i}, \widehat{P}_{j}\right)$, is given by

$$
\left\{X_{i}, P_{j}\right\}=\delta_{i j} \Rightarrow\left[\widehat{X}_{i}, \widehat{P}_{j}\right]=i \hbar \delta_{i j}
$$

In case we know a classical invariant of the system, we can easily identify the counterpart quantum invariant on account of the above simple relation. By replacing the canonical variables in the classical invariant with the corresponding quantum operators, we have the quantum invariant operator such that

$$
\begin{aligned}
O(t)= & \frac{1}{2} \sum_{i=1}^{3}\left[\frac{\widehat{P}_{i}^{2}}{m_{i}(t)}+\frac{\dot{m}_{i}(t)}{m_{i}(t)}\left(\widehat{X}_{i} \widehat{P}_{i}+\widehat{X}_{i} \widehat{P}_{i}\right)+\left(\int_{0}^{t} \omega_{i}^{2}(t) \dot{m}_{i}(t) d t\right) \widehat{X}_{i}^{2}\right] \\
& +\frac{1}{2}\left(\int_{0}^{t} k_{12}(t)\left(\frac{\dot{m}_{1}(t)}{2 m_{1}(t)}+\frac{\dot{m}_{2}(t)}{2 m_{2}(t)}\right) d t\right) \widehat{X}_{1} \widehat{X}_{2} \\
& +\frac{1}{2}\left(\int_{0}^{t} k_{13}(t)\left(\frac{\dot{m}_{1}(t)}{2 m_{1}(t)}+\frac{\dot{m}_{3}(t)}{2 m_{3}(t)}\right) d t\right) \widehat{X}_{1} \widehat{X}_{3} \\
& +\frac{1}{2}\left(\int_{0}^{t} k_{23}(t)\left(\frac{\dot{m}_{2}(t)}{2 m_{2}(t)}+\frac{\dot{m}_{3}(t)}{2 m_{3}(t)}\right) d t\right) \widehat{X}_{2} \widehat{X}_{3} .
\end{aligned}
$$

We can show that this invariant obeys the Liouville-von Neumann equation which is of the form

$$
\frac{d \widehat{O}(t)}{d t}=\frac{\partial \widehat{O}(t)}{\partial t}+\frac{1}{i \hbar}[\widehat{O}(t), \widehat{H}(t)]=0
$$

Because the quantum invariant operator given in Equation (22) is a somewhat complicated form, it may be necessary to simplify it forits use in the dynamical analysis of the system. For this purpose, we will use the unitary transformation method by introducing appropriate unitary operators.

At first, we consider the following unitary operator

$$
\widehat{U}(t)=\widehat{U}_{1}(t) \widehat{U}_{2}(t)
$$

where $\widehat{U}_{1}(t)$ and $\widehat{U}_{2}(t)$ are given by

$$
\begin{aligned}
& \widehat{U}_{1}(t)=\prod_{i=1}^{3} \exp \left[\frac{1}{2 \hbar}\left(\widehat{P}_{i} \widehat{X}_{i}+\widehat{X}_{i} \widehat{P}_{i}\right) \ln \sqrt{m_{i}(t)}\right] \\
& \widehat{U}_{2}(t)=\exp \left[-\frac{i}{4 \hbar} \sum_{i=1}^{3} \frac{\dot{m}_{i}(t)}{m_{i}(t)} \widehat{X}_{i}^{2}\right] .
\end{aligned}
$$

Using this operator, we transform the invariant $\widehat{O}(t)$ as

$$
\widehat{\mathscr{O}}(t)=U \wedge^{-1}(t) \widehat{O}(t) \widehat{U}(t)
$$

Then, after a straightforward evaluation, we get

$$
\begin{aligned}
\widehat{\mathcal{O}}(t)= & \frac{1}{2} \sum_{i=1}^{3}\left[\widehat{P}_{i}^{2}+\left(\frac{\int_{0}^{t} \omega_{i}^{2}(t) \dot{m}_{i}(t) d t}{m_{i}(t)}-\left[\frac{\dot{m}_{i}(t)}{4 m_{i}(t)}\right]^{2}\right) \widehat{X}_{i}^{2}\right] \\
& +\frac{1}{2}\left(\frac{\int_{0}^{t} k_{12}(t)\left(\left(\dot{m}_{1}(t) / m_{1}(t)\right)+\left(\dot{m}_{2}(t) / m_{2}(t)\right)\right) d t}{\sqrt{m_{1}(t) m_{2}(t)}}\right) \widehat{X}_{1} \widehat{X}_{2} \\
& +\frac{1}{2}\left(\frac{\int_{0}^{t} k_{13}(t)\left(\left(\dot{m}_{1}(t) / m_{1}(t)\right)+\left(\dot{m}_{3}(t) / m_{3}(t)\right)\right) d t}{\sqrt{m_{1}(t) m_{3}(t)}}\right) \widehat{X}_{1} \widehat{X}_{3} \\
& +\frac{1}{2}\left(\frac{\int_{0}^{t} k_{23}(t)\left(\left(\dot{m}_{2}(t) / m_{2}(t)\right)+\left(\dot{m}_{3}(t) / m_{3}(t)\right)\right) d t}{\sqrt{m_{2}(t) m_{3}(t)}}\right) \widehat{X}_{2} \widehat{X}_{3} .
\end{aligned}
$$

We see that the coefficients $m_{i}^{-1}$ of $\widehat{P}_{i}^{2}$ terms in the original invariant operator have been eliminated through the above transformation. However, $\widehat{\mathcal{O}}(t)$ involves interacting 
terms $\widehat{X}_{i} \widehat{X}_{j}$ yet. For further simplification of the invariant operator with the removal of the interacting terms, we consider the diagonalization of its matrix representation in the subsequent subsection.

2.3. Diagonalization of the Invariant Operator. Removing of the interacting terms in Equation (28) may be very helpful for evaluating, for example, the eigenfunctions of the invariant operator. Such a removal can be fulfilled by diagonalizing the invariant operator after converting it into an equivalent matrix. We easily confirm that the matrix form of Equation (28) is given by

$$
\widehat{\mathcal{O}}(t)=\frac{1}{2} \sum_{i, j=1}^{3} \widehat{P}_{i} \delta_{i j} \widehat{P}_{j}+\frac{1}{2} \sum_{i, j=1}^{3} \widehat{X}_{i} \mathbb{k}_{i j} \widehat{X}_{j}
$$

where $\delta_{i j}=1$ for $i=j$ while $\delta_{i j}=0$ otherwise, $\mathbb{k}_{i j}$ are $i$ th row and $j$ th column elements of the matrix $\mathbb{k}$, and the two matrices are represented as

$$
\mathbb{k}=\left(\begin{array}{ccc}
\omega_{1}^{2} & \frac{1}{2} K_{12} & \frac{1}{2} K_{13} \\
\frac{1}{2} K_{12} & \omega_{2}^{2} & \frac{1}{2} K_{23} \\
\frac{1}{2} K_{13} & \frac{1}{2} K_{23} & \omega_{3}^{2}
\end{array}\right), \quad X=\left(\begin{array}{c}
X_{1} \\
X_{2} \\
X_{3}
\end{array}\right),
$$

whereas the involved parameters are given by

$$
\begin{aligned}
\omega_{i} & =\left(\frac{\int_{0}^{t} \omega_{i}^{2}(t) \dot{m}_{i}(t) d t}{m_{i}(t)}-\left[\frac{\dot{m}_{i}(t)}{4 m_{i}(t)}\right]^{2}\right)^{1 / 2}, \\
K_{12} & =\frac{\int_{0}^{t} k_{12}(t)\left(\left(\dot{m}_{1}(t) / m_{1}(t)\right)+\left(\dot{m}_{2}(t) / m_{2}(t)\right)\right) d t}{\sqrt{m_{1}(t) m_{2}(t)}}, \\
K_{13} & =\frac{\int_{0}^{t} k_{13}(t)\left(\left(\dot{m}_{1}(t) / m_{1}(t)\right)+\left(\dot{m}_{3}(t) / m_{3}(t)\right)\right) d t}{\sqrt{m_{1}(t) m_{3}(t)}}, \\
K_{23} & =\frac{\int_{0}^{t} k_{23}(t)\left(\left(\dot{m}_{2}(t) / m_{2}(t)\right)+\left(\dot{m}_{3}(t) / m_{3}(t)\right)\right) d t}{\sqrt{m_{2}(t) m_{3}(t)}} .
\end{aligned}
$$

It is known that not only a diagonal but a diagonalizable matrixes are square matrixes as well $[30,31]$. In general, this property is equivalent to the existence of a basis of eigenvectors and makes it possible to define in a diagonalizable endomorphism in a vector space. Therefore, to diagonalize the first matrix in Equation (30), it is necessary to seek square forms of its eigenvalues, $\Omega_{i}^{2}$, and the corresponding eigenvectors $\vec{V}_{i}$. Hence the diagonalized representation of $\mathbb{k}$ can be represented in the form

$$
\mathbb{R}^{-1} \mathbb{k} \mathbb{R}\left(\begin{array}{ccc}
\Omega_{1}^{2} & 0 & 0 \\
0 & \Omega_{2}^{2} & 0 \\
0 & 0 & \Omega_{3}^{2}
\end{array}\right) .
$$

We will evaluate $\Omega_{i}^{2}$ together with the eigenvectors $\vec{V}_{i}$ from now on. For this, we consider the eigenvalue equation

$$
\left(\mathbb{k}-\Omega_{i}^{2} I\right) \vec{V}_{i}=0,
$$

where $I$ is the identity matrix. Considering that the eigenvalues of the matrix $\mathrm{k}$ are values of $\alpha$, we represent the secular equation as

$$
|\mathbb{k}-\alpha I|=\left(\Omega_{1}^{2}-\alpha\right)\left(\Omega_{2}^{2}-\alpha\right)\left(\Omega_{3}^{2}-\alpha\right)=0 .
$$

Then, the eigenvalues of the matrix $\mathbb{k}$ are obtained to be

$$
\begin{aligned}
& \Omega_{1}^{2}=\omega_{1}^{2}+\frac{K_{12}+K_{13}}{2}, \\
& \Omega_{2}^{2}=\omega_{2}^{2}-\frac{1}{2} K_{23}+\Omega^{2}, \\
& \Omega_{3}^{2}=\omega_{3}^{2}-\frac{1}{2} K_{23}-\Omega^{2},
\end{aligned}
$$

where

$$
\Omega^{2}=\frac{1}{2}\left[K_{12}^{2}+K_{13}^{2}+K_{23}^{2}-\left(K_{12} K_{23}+K_{12} K_{23}+K_{13} K_{23}\right)\right]^{1 / 2} .
$$

From a straightforward evaluation, each eigenvector related to $\Omega_{i}^{2}$ is given by

$$
\begin{aligned}
& \vec{V}_{1}=\frac{1}{\sqrt{3}}\left(\begin{array}{c}
1 \\
1 \\
1
\end{array}\right), \\
& \vec{V}_{2}=\lambda_{+}\left(\begin{array}{c}
\frac{1}{2} K_{12}-\frac{1}{2} K_{23}-\Omega^{2} \\
\frac{1}{2} K_{23}-\frac{1}{3} K_{12}+\Omega^{2} \\
\frac{1}{2} K_{23}-\frac{1}{2} K_{13}
\end{array}\right), \\
& \vec{V}_{3}=\lambda_{-}\left(\begin{array}{c}
\frac{1}{2} K_{12}-\frac{1}{2} K_{23}+\Omega^{2} \\
\frac{1}{2} K_{23}-\frac{1}{2} K_{12}-\Omega^{2} \\
\frac{1}{2} K_{23}-\frac{1}{2} K_{13}
\end{array}\right),
\end{aligned}
$$


where

$$
\lambda_{ \pm}=\frac{1}{K_{23}-K_{13}}\left[\frac{2}{3} \pm \frac{K_{12}-\left(K_{13}+K_{23}\right) / 2}{3 \Omega^{2}}\right]^{1 / 2} .
$$

Similar eigenvectors are introduced in Ref. [14] but for a Hamiltonian instead of the invariant. On the other hand, the matrix form of $\mathbb{R}$ is written as a block of the column vectors

$$
\mathbb{R}=\left(\vec{V}_{1} \vec{V}_{2} \vec{V}_{3}\right)=\left(\begin{array}{ccc}
\frac{1}{\sqrt{3}} & \lambda_{+}\left[\frac{1}{2} K_{12}-\frac{1}{2} K_{23}-\Omega^{2}\right] & \lambda_{-}\left[\frac{1}{2} K_{12}-\frac{1}{2} K_{23}+\Omega^{2}\right] \\
\frac{1}{\sqrt{3}} & \lambda_{+}\left[-\frac{1}{2} K_{12}+\frac{1}{2} K_{23}+\Omega^{2}\right] & \lambda_{-}\left[-\frac{1}{2} K_{12}+\frac{1}{2} K_{23}-\Omega^{2}\right] \\
\frac{1}{\sqrt{3}} & \lambda_{+}\left[-\frac{1}{2} K_{13}+\frac{1}{2} K_{23}\right] & \lambda_{-}\left[-\frac{1}{2} K_{13}+\frac{1}{2} K_{23}\right]
\end{array}\right) .
$$

Now, in terms of the new coordinates

$$
\left(\begin{array}{l}
x_{1} \\
x_{2} \\
x_{3}
\end{array}\right)=\mathbb{R}\left(\begin{array}{l}
X_{1} \\
X_{2} \\
X_{3}
\end{array}\right),\left(\begin{array}{l}
p_{1} \\
p_{2} \\
p_{3}
\end{array}\right)=\mathbb{R}\left(\begin{array}{c}
P_{1} \\
P_{2} \\
P_{3}
\end{array}\right),
$$

the diagonalized invariant operator $\widehat{\widehat{O}}$ takes the form

$$
\widehat{\mathcal{O}}=\frac{1}{2} \sum_{i=1}^{3} \frac{1}{2}\left(\hat{p}_{i}^{2}+\frac{1}{2} \Omega_{i}^{2} \widehat{x}_{i}^{2}\right) .
$$

Here, the full expressions of $\hat{x}_{i}$ are given by

$\widehat{x}_{1}=\frac{1}{\sqrt{3}} \widehat{X}_{1}+\frac{\lambda_{+}}{2}\left(K_{12}-K_{23}-2 \Omega^{2}\right) \widehat{X}_{2}+\frac{\lambda_{-}}{2}\left(K_{12}-K_{23}+2 \Omega^{2}\right) \widehat{X}_{3}$,

$\widehat{x}_{2}=\frac{1}{\sqrt{3}} \widehat{X}_{1}+\frac{\lambda_{+}}{2}\left(K_{23}-K_{12}+2 \Omega^{2}\right) \widehat{X}_{2}+\frac{\lambda_{-}}{2}\left(K_{23}-K_{12}-2 \Omega^{2}\right) \widehat{X}_{3}$,

$\widehat{x}_{3}=\frac{1}{\sqrt{3}} \hat{X}_{1}+\frac{\lambda_{+}}{2}\left(K_{23}-K_{13}\right) \hat{X}_{2}+\frac{\lambda_{-}}{2}\left(K_{23}-K_{13}\right) \hat{X}_{3}$.

$\widehat{p}_{i}$ are also represented in the same manners. Equation (48) is a sum of three simple harmonic oscillators with constant frequencies and unit masses. We can easily manage the diagonalized invariant due to its simplicity. For instance, it is possible to solve the eigenvalue equation of it without difficulty. This means that the diagonalized invariant can be usefully applied to analyzing various dynamical properties of the system. In the subsequent section, we will apply the diagonalized invariant in the investigation of quantum characteristics of the system.

\section{Application in Quantum Mechanics}

Until now, we have constructed an invariant operator for the three coupled nano-optomechanical oscillators and diago- nalized it. We can easily find the solutions of the eigenvalue equation of the diagonalized invariant operator. We will apply this, in this section, in the quantum mechanical problem of the system as an example. Exact wave functions of the system will be derived by taking advantage of the eigenfunctions of the invariant operator.

3.1. Eigenfunctions. For quantum mechanical description of the nano-optomechanical system, it may be necessary to see the eigenfunctions of the invariant operator because they are closely related to the quantum wave functions. More clearly speaking, quantum wave functions for time-varying oscillatory systems are represented in terms of the eigenfunctions according to the invariant operator theory $[15,16]$.

Equation (48) is the same as the Hamiltonian associated to the three independent oscillators. Hence, the eigenfunctions of the diagonalized invariant operator are simply the same as those of the Hamiltonian for the uncoupled 3D oscillator. From the inverse transformation of the well-known form of such eigenfunctions, it may be possible to obtain the eigenfunctions of the original invariant operator. Through a rigorous mathematical procedure, we will demonstrate it in this section. In addition, we will also show that the wave functions of the system can be obtained by using such eigenfunctions.

In order to obtain the eigenvalues of the invariant operator $\widehat{O}(t)$, we first introduce the canonical annihilation and creation operators $\widehat{b}_{i}$ and $\widehat{b}_{i}^{\dagger}$ associated to the transformed invariant operator:

$$
\begin{aligned}
& \widehat{b}_{i}=\frac{1}{\sqrt{2}}\left(\sqrt{\Omega_{i}} \widehat{x}_{i}+\frac{i}{\sqrt{\Omega_{i}}} \widehat{p}_{i}\right), \\
& \widehat{b}_{i}^{\dagger}=\frac{1}{\sqrt{2}}\left(\sqrt{\Omega_{i}} \widehat{x}_{i}-\frac{i}{\sqrt{\Omega_{i}}} \widehat{p}_{i}\right) .
\end{aligned}
$$

Notice that these operators obey the usual boson 
canonical commutation rule, which is $\left[\widehat{b}_{i}, \hat{b}_{i}^{\dagger}\right]=1$. Then, Equation (48) can be expressed in terms of $\widehat{b}_{i}$ and $\widehat{b}_{i}^{\dagger}$ as

$$
\widehat{\mathcal{O}}=\sum_{i=1}^{3} \hbar \Omega_{i}\left(\widehat{b}_{i}^{\dagger} \widehat{b}_{i}+\frac{1}{2}\right)
$$

We now consider the inverse unitary transformation:

$$
\widehat{O}(t)=\widehat{U}(t) \widehat{\sigma} U \wedge^{-1}(t), \quad \widehat{a}_{i}(t)=\widehat{U}(t) \widehat{b}_{i} U \Lambda^{-1}(t), \quad \widehat{a}_{i}^{\dagger}(t)=\widehat{U}(t) \hat{b}_{i}^{\dagger} U \Lambda^{-1}(t) .
$$

where $\widehat{a}_{i}(t)$ and $\widehat{a}_{i}^{\dagger}(t)$ are the time-dependent canonical annihilation and creation operators associated with the original invariant. Note that this transformation changes $\widehat{X}_{i}$ and $\widehat{P}_{i}$ to be

$$
\begin{gathered}
\widehat{X}_{i} \longrightarrow \widehat{U}(t) \widehat{X}_{i} U \wedge^{-1}(t)=\sqrt{m_{i}(t)} \widehat{X} \\
\widehat{P}_{i} \longrightarrow \widehat{U}(t) \widehat{P}_{i} U \wedge^{-1}(t)=\frac{1}{\sqrt{m_{i}(t)}}\left(\widehat{P}_{i}+\frac{\dot{m}_{i}(t)}{2} \widehat{X}_{i}\right) .
\end{gathered}
$$

Thanks to this, the invariant operator $\widehat{O}(t)$, Equation (20), can be rewritten in the form

$$
\widehat{O}(t)=\sum_{i=1}^{3} \hbar \Omega_{i}\left[\widehat{a}_{i}^{\dagger}(t) \widehat{a}_{i}(t)+\frac{1}{2}\right],
$$

whereas the full expressions of $\widehat{a}_{i}(t)$ and $\hat{a}_{i}^{\dagger}(t)$ are given by

$$
\begin{aligned}
& \widehat{a}_{1}(t)=\left(\frac{\Omega_{1}}{2}\right)^{1 / 2} \widehat{X}_{+}+i\left(\frac{1}{2 \Omega_{1}}\right)^{1 / 2} \widehat{P}_{+}, \\
& \widehat{a}_{1}^{\dagger}(t)=\left(\frac{\Omega_{1}}{2}\right)^{1 / 2} \widehat{X}_{+}-i\left(\frac{1}{2 \Omega_{1}}\right)^{1 / 2} \widehat{P}_{+}, \\
& \widehat{a}_{2}(t)=\left(\frac{\Omega_{2}}{2}\right)^{1 / 2} \widehat{X}_{-}+i\left(\frac{1}{2 \Omega_{2}}\right)^{1 / 2} \widehat{P}_{-}, \\
& \widehat{a}_{2}^{\dagger}(t)=\left(\frac{\Omega_{2}}{2}\right)^{1 / 2} \widehat{X}_{-}-i\left(\frac{1}{2 \Omega_{2}}\right)^{1 / 2} \widehat{P}_{-}, \\
& \widehat{a}_{3}(t)=\left(\frac{\Omega_{3}}{2}\right)^{1 / 2} \widehat{X}_{0}+i\left(\frac{1}{2 \Omega_{3}}\right)^{1 / 2} \widehat{P}_{0}, \\
& \widehat{a}_{3}^{\dagger}(t)=\left(\frac{\Omega_{3}}{2}\right)^{1 / 2} \widehat{X}_{0}-i\left(\frac{1}{2 \Omega_{3}}\right)^{1 / 2} \widehat{P}_{0},
\end{aligned}
$$

with

$$
\begin{aligned}
\widehat{X}_{ \pm}= & \frac{1}{\sqrt{3}} \sqrt{m_{1}(t)} \hat{X}_{1} \pm \frac{\lambda_{+}}{2}\left(K_{12}-K_{23}-2 \Omega^{2}\right) \sqrt{m_{2}(t)} \widehat{X}_{2} \\
& \pm \frac{\lambda_{-}}{2}\left(K_{12}-K_{23}+2 \Omega^{2}\right) \sqrt{m_{3}(t)} \widehat{X}_{3},
\end{aligned}
$$

$$
\begin{aligned}
\widehat{P}_{ \pm}= & \frac{1}{\sqrt{3}} \frac{1}{\sqrt{m_{1}(t)}}\left(\widehat{P}_{1}+\frac{\dot{m}_{1}(t)}{2} \widehat{X}_{1}\right) \\
& \pm \frac{\lambda_{+}}{2}\left(K_{12}-K_{23}-2 \Omega^{2}\right) \frac{1}{\sqrt{m_{2}(t)}}\left(\widehat{P}_{2}+\frac{\dot{m}_{2}(t)}{2} \widehat{X}_{2}\right) \\
& \pm \frac{\lambda_{-}}{2}\left(K_{12}-K_{23}+2 \Omega^{2}\right) \frac{1}{\sqrt{m_{3}(t)}}\left(\widehat{P}_{3}+\frac{\dot{m}_{3}(t)}{2} \widehat{X}_{3}\right)
\end{aligned}
$$

$$
\begin{aligned}
\widehat{X}_{0}= & \frac{1}{\sqrt{3}} \sqrt{m_{1}(t)} \widehat{X}_{1}+\frac{\lambda_{+}}{2}\left(K_{23}-K_{13}\right) \sqrt{m_{2}(t)} \widehat{X}_{2} \\
& +\frac{\lambda_{-}}{2}\left(K_{23}-K_{13}\right) \sqrt{m_{3}(t)} \widehat{X}_{3}, \\
\widehat{P}_{0}= & \frac{1}{\sqrt{3}} \frac{1}{\sqrt{m_{1}(t)}}\left(\widehat{P}_{1}+\frac{\dot{m}_{1}(t)}{2} \widehat{X}_{1}\right) \\
& +\frac{\lambda_{+}}{2}\left(K_{23}-K_{13}\right) \frac{1}{\sqrt{m_{2}(t)}}\left(\widehat{P}_{2}+\frac{\dot{m}_{2}(t)}{2} \widehat{X}_{2}\right) \\
& +\frac{\lambda_{-}}{2}\left(K_{23}-K_{13}\right) \frac{1}{\sqrt{m_{3}(t)}}\left(\widehat{P}_{3}+\frac{\dot{m}_{3}(t)}{2} \widehat{X}_{3}\right) .
\end{aligned}
$$

Let us denote the eigenstates of $\widehat{a}_{i}^{\dagger} \widehat{a}_{i}$ as $\left|n_{i}, t\right\rangle$. Then, the eigenvalue equations of the number operators are given by

$$
\widehat{a}_{i}^{\dagger} \widehat{a}_{i}\left|n_{i}, t\right\rangle=n_{i}\left|n_{i}, t\right\rangle,
$$

where $n_{i}=0,1,2, \cdots$. We now easily confirm that the eigenvalues of Equation (58) are represented in terms of $n_{i}$ as

$$
\lambda_{n_{1}, n_{2}, n_{3}}=\sum_{i=1}^{3} \hbar \Omega_{i}\left(n_{i}+\frac{1}{2}\right) .
$$

The complete eigenstates of $\widehat{O}$ can be written as a product of three individual eigenstates that can be evaluated from the zero-point eigenstates:

$$
\begin{aligned}
& \left|n_{1}, n_{2}, n_{3}, t\right\rangle=\left|n_{1}, t\right\rangle \otimes\left|n_{2}, t\right\rangle \otimes\left|n_{3}, t\right\rangle \\
& \quad=\frac{1}{\sqrt{n_{1} ! n_{2} ! n_{3} !}}\left(a \wedge_{1}^{\dagger}\right)^{n_{1}}\left(a \wedge_{2}^{\dagger}\right)^{n_{2}}\left(a \wedge_{3}^{\dagger}\right)^{n_{3}}|0,0,0, t\rangle .
\end{aligned}
$$

According to the general convention, we choose the amplitudes of $\left|n_{1}, n_{2}, n_{3}, t\right\rangle$ in a way that the states obey the normalization condition, i.e.,

$$
\left\langle n_{1}, n_{2}, n_{3}, t \mid n_{1}^{\prime}, n_{2}^{\prime}, n_{3}^{\prime}, t\right\rangle=\delta_{n_{1}, n_{1}^{\prime}} \delta_{n_{2}, n_{2}^{\prime}} \delta_{n_{3}, n_{3}^{\prime}}
$$

The eigenstates in Equation (71) are necessary in unfolding quantum theory of the system.

3.2. Wave Functions. According to the Lewis-Riesenfeld theory, the wave functions $\left|\psi_{n_{1}, n_{2}, n_{3}}(t)\right\rangle$ of the nanooptomechanical system are represented in terms of the eigenstates derived in the previous section: 


$$
\left|\psi_{n_{1}, n_{2}, n_{3}}(t)\right\rangle=e^{i \zeta_{n_{1}, n_{2}, n_{3}}(t)}\left|n_{1}, n_{2}, n_{3}, t\right\rangle
$$

where $\zeta_{n_{1}, n_{2}, n_{3}}(t)$ are phases. We can determine $\zeta_{n_{1}, n_{2}, n_{3}}(t)$ from the Schrödinger equation, which is of the form

$$
i \hbar \frac{\partial}{\partial t}\left|\psi_{n_{1}, n_{2}, n_{3}}(t)\right\rangle=\widehat{H}\left|\psi_{n_{1}, n_{2}, n_{3}}(t)\right\rangle
$$

A minor evaluation after inserting Equation (73) into the above equation leads to

$$
\frac{\partial}{\partial t} \zeta_{n_{1}, n_{2}, n_{3}}(t)=\frac{1}{\hbar}\left\langle n_{1}, n_{2}, n_{3}, t\left|\left(\frac{\partial}{\partial t}-\widehat{H}\right)\right| n_{1}, n_{2}, n_{3}, t\right\rangle .
$$

By solving this equation in a straightforward way, we have

$$
\zeta_{n_{1}, n_{2}, n_{3}}(t)=\sum_{i=1}^{3} \Omega_{i}\left(n_{i}+\frac{1}{2}\right) t
$$

Finally, the wave functions in the configuration space are written in the form

$$
\begin{aligned}
& \left\langle\widehat{X}_{1}, \widehat{X}_{2}, \widehat{X}_{3} \mid \psi_{n_{1}, n_{2}, n_{3}}(t)\right\rangle=\prod_{i=1}^{3}\left[\left(\frac{\sqrt{\Omega_{i} m_{i}(t)}}{(\pi \hbar)^{1 / 2} n_{i} ! 2^{n_{i}}}\right)^{1 / 2} H_{n_{i}}\left(\mu_{i}\right)\right] \\
& \times \exp \left\{-\sum_{i=1}^{3}\left[\left(\frac{\Omega_{i}}{2 \hbar}+\frac{i \dot{m}_{i}(t)}{4 \hbar m_{i}(t)}\right) \sum_{j=1}^{3}\left(\mathbb{R}_{i j} \sqrt{m_{j}(t)} X_{j}\right)^{2}\right]\right\} \\
& \quad \times \exp \left[-i \sum_{i=1}^{3} \Omega_{i}\left(n_{i}+\frac{1}{2}\right) t\right],
\end{aligned}
$$

where

$$
\begin{aligned}
\mu_{1}= & \left(\frac{\Omega_{1}}{\hbar}\right)^{1 / 2}\left[\frac{1}{\sqrt{3}} \sqrt{m_{1}(t)} X_{1}+\frac{\lambda_{+}}{2}\left(K_{12}-K_{23}-2 \Omega^{2}\right) \sqrt{m_{2}(t)} X_{2}\right. \\
& \left.+\frac{\lambda_{-}}{2}\left(K_{12}-K_{23}+2 \Omega^{2}\right) \sqrt{m_{3}(t)} X_{3}\right]
\end{aligned}
$$

$$
\begin{aligned}
\mu_{2}= & \left(\frac{\Omega_{2}}{\hbar}\right)^{1 / 2}\left[\frac{1}{\sqrt{3}} \sqrt{m_{1}(t)} X_{1}+\frac{\lambda_{+}}{2}\left(K_{23}-K_{12}+2 \Omega^{2}\right) \sqrt{m_{2}(t)} X_{2}\right. \\
& \left.+\frac{\lambda_{-}}{2}\left(K_{23}-K_{12}-2 \Omega^{2}\right) \sqrt{m_{3}(t)} X_{3}\right]
\end{aligned}
$$

$$
\begin{aligned}
\mu_{3}= & \left(\frac{\Omega_{3}}{\hbar}\right)^{1 / 2}\left[\frac{1}{\sqrt{3}} \sqrt{m_{1}(t)} X_{1}+\frac{\lambda_{+}}{2}\left(K_{23}-K_{13}\right) \sqrt{m_{2}(t)} X_{2}\right. \\
& \left.+\frac{\lambda_{-}}{2}\left(K_{23}-K_{13}\right) \sqrt{m_{3}(t)} X_{3}\right] .
\end{aligned}
$$

Notice that $\mu_{i}$ are not only functions of position variables $X_{i}$, but vary depending on time as well.

Although the full wave functions, Equation (77), are somewhat complicated, they play basic tool for evaluating not only the quantum mechanical expectation values of various observables such as physical momentum and quantum energy but also the probability distribution, the entanglement of systems, and the propagator. Hence, we can characterize various quantum properties associated with the system by means of the wave functions.

\section{Conclusion}

The invariant operator of time-dependent three coupled nano-optomechanical oscillators was investigated. Starting from the formulation of the classical invariant, we have obtained the quantum invariant operator of the system. It was shown that the invariant operator obeys the Liouvillevon Neumann equation.

We also investigated the diagonalization of the invariant operator in order to show the usefulness of the quantum invariant in analyzing the dynamical properties of the nano-optomechanical system. As a preliminary step before the practical diagonalization of the invariant operator, we have carried out the unitary transformation of it; this procedure allowed us to transform the quantal invariant operator to an equal, but a simple one, which is represented by unit masses.

However, the transformed invariant operator still involve the coupling terms $\widehat{X}_{i} \widehat{X}_{j}$. Such terms were eliminated by the eventual diagonalization of the matrix representation of the invariant.

The diagonalized invariant is no longer a function of time and it can be represented simply in terms of the creation and annihilation operators $\left(\widehat{b}_{i}^{\dagger}, \widehat{b}_{i}\right)$ (see Equation (54)). Hence, by virtue of its diagonalization, the invariant can be effectively used in analyzing diverse dynamical properties of the nanooptomechanical oscillatory systems. For instance, we can easily identify the eigenstates of the original invariant operator based on fundamental unitary relations. With the help of these eigenstates, the wave functions satisfying the Schrödinger equation have been obtained as shown in Equation (77). Apparently, this shows the powerfulness of the invariant operator when we treat the nano-optomechanical system quantum mechanically. If we think of the fact that quantum characteristics of a mechanical system can be usually examined starting from the interpretation of wave functions, the wave functions obtained here may open a way for clarifying quantum effects of the nano-optomechanical system from fundamental mathematical point of view. This is the main contribution of our research in this work.

In particular, entanglement dynamics between elements of the three or multicoupled nano-optomechnical oscillators can be investigated based on our research. The creation and control of entanglement in coupled quantum optomechanical oscillators are crucial in the realization of quantum information processing in quantum cryptography and quantum computing [32]. Because the technologies related to them 
utilize novel nonlocal quantum effects, clarification of fundamental quantum characteristics of the system is necessary. Notably, our quantum formalism in this context based on the dynamical invariant does not require any approximation and perturbation method even when the parameters vary adiabatically in time.

We know that the size of optomechanical and electromechanical systems becomes gradually smaller from day to day according to the rapid advance of nanotechnology. As the size of optomechanical devices reaches nanoscale, classical mechanics can no longer be applicable in describing mechanical properties of the systems. This is due to the prominence of quantum effects in the realm of nanoscale devices [33-36]. Hence, we should inevitably use quantum mechanics in order to analyze the dynamics of nano-optomechanical oscillatory systems. The feasibility of quantum description of nanooptomechanical systems based on invariant operator theory that we have treated here may provide an actual solution along this line.

\section{Data Availability}

The data used to support the findings of this study are included within the article.

\section{Conflicts of Interest}

The authors declare that they have no conflicts of interest.

\section{Acknowledgments}

This work was supported by the National Research Foundation of Korea (NRF) grant funded by the Korea Government (MSIT) (No.: NRF-2021R1F1A1062849).

\section{References}

[1] M. Ebert, A. Volosniev, and H. W. Hammer, "Two cold atoms in a time-dependent harmonic trap in one dimension," Annalen der Physik, vol. 528, no. 9-10, pp. 693-704, 2016.

[2] J. A. González, J. J. Suárez-Vargas, A. Stefanovska, and P. V. E. McClintock, "Fluctuations in a coupled-oscillator model of the cardiovascular system," Proceedings of SPIE, vol. 6602, p. 66020Y, 2007.

[3] G. Csaba and W. Porod, "Coupled oscillators for computing: a review and perspective," Applied Physics Reviews, vol. 7, no. 1, p. $011302,2020$.

[4] C. J. Wilson and J. C. Callaway, "Coupled oscillator model of the dopaminergic neuron of the substantia nigra," Journal of Neurophysiology, vol. 83, no. 5, pp. 3084-3100, 2000.

[5] A. Collado and A. Georgiadis, "Coupled oscillator systems for microwave applications: optimized design based on the study and control of the multiple coexisting solutions in systems with symmetry," in Recent Advances in Nonlinear Dynamics and Synchronization. Studies in Computational Intelligence, K. Kyamakya, W. A. Halang, H. Unger, J. C. Chedjou, N. F. Rulkov, and Z. Li, Eds., vol. 254, Springer, Berlin, Heidelberg, 2009.

[6] E. Gil-Santos, M. Labousse, C. Baker et al., "Light-mediated cascaded locking of multiple nano-optomechanical oscillators," Physical Review Letters, vol. 118, no. 6, p. 063605, 2017.
[7] N. Spethmann, J. Kohler, S. Schreppler, L. Buchmann, and D. M. Stamper-Kurn, "Cavity-mediated coupling of mechanical oscillators limited by quantum back- action," Nature Physics, vol. 12, no. 1, pp. 27-31, 2016.

[8] A. G. Litvak and M. D. Tokman, "Electromagnetically induced transparency in ensembles of classical oscillators," Physical Review Letters, vol. 88, no. 9, p. 095003, 2002.

[9] P. R. Hemmer and M. G. Prentiss, "Coupled-pendulum model of the stimulated resonance Raman effect," Journal of the Optical Society of America B: Optical Physics, vol. 5, no. 8, pp. 1613$1623,1988$.

[10] D. B. Sullivan and J. E. Zimmerman, "Mechanical analogs of time dependent Josephson phenomena," American Journal of Physics, vol. 39, no. 12, pp. 1504-1517, 1971.

[11] R. Marx and S. J. Glaser, "Spins swing like pendulums do: an exact classical model for TOCSY transfer in systems of three isotropically coupled spins 1/2," Journal of Magnetic Resonance, vol. 164, no. 2, pp. 338-342, 2003.

[12] A. Merdaci and A. Jellal, "Entanglement in three coupled harmonic oscillators," Physics Letters A, vol. 384, no. 6, p. 126134, 2019.

[13] D. Park, "Dynamics of entanglement and uncertainty relation in coupled harmonic oscillator system: exact results," Quantum Information Processing, vol. 17, no. 6, p. 147, 2018.

[14] D. Park, "Dynamics of entanglement in three coupled harmonic oscillator system with arbitrary time-dependent frequency and coupling constants," Quantum Information Processing, vol. 18, no. 9, p. 282, 2019.

[15] H. R. Lewis Jr., "Class of exact invariants for classical and quantum time-dependent harmonic oscillators," Journal of Mathematical Physics, vol. 9, no. 11, pp. 1976-1986, 1968.

[16] H. R. Lewis Jr. and W. B. Riesenfeld, "An exact quantum theory of the time-dependent harmonic oscillator and of a charged particle in a time-dependent electromagnetic field," Journal of Mathematical Physics, vol. 10, no. 8, pp. 14581473, 1969.

[17] S. Menouar, M. Maamache, and J. R. Choi, "The timedependent coupled oscillator model for the motion of a charged particle in the presence of a time-varying magnetic field," Physica Scripta, vol. 82, no. 6, p. 065004, 2010.

[18] S. Menouar, M. Maamache, and J. R. Choi, "An alternative approach to exact wave functions for time-dependent coupled oscillator model of charged particle in variable magnetic field," Annals of Physics, vol. 325, no. 8, pp. 1708-1719, 2010.

[19] S. L. Wu, X. Y. Zhang, and X. X. Yi, "Dynamical invariants of open quantum systems," Physical Review A, vol. 92, no. 6, p. $062122,2015$.

[20] A. Levy, A. Kiely, J. G. Muga, R. Kosloff, and E. Torrontegui, "Noise resistant quantum control using dynamical invariants," New Journal of Physics, vol. 20, no. 2, p. 025006, 2018.

[21] M. C. Bertin, B. M. Pimentel, and J. A. Ramirez, "Construction of time-dependent dynamical invariants: a new approach," Journal of Mathematical Physics, vol. 53, no. 4, p. 042104, 2012.

[22] L. Billings and I. B. Schwartz, "Identifying almost invariant sets in stochastic dynamical systems," Chaos, vol. 18, no. 2, p. $023122,2008$.

[23] Z. Shi and S. Abe, "Quantum weak invariants: dynamical evolution of fluctuations and correlations," Entropy, vol. 22, no. 11, p. 1219, 2020.

[24] S. K. Suslov, "Dynamical invariants for variable quadratic Hamiltonians," Physica Scripta, vol. 81, no. 5, p. 055006, 2010. 
[25] S. Zhang, J. R. Choi, C. I. Um, and K. H. Yeon, "Quantum uncertainties of mesoscopic capacitance coupled circuit," Physics Letters A, vol. 289, no. 4-5, pp. 257-263, 2001.

[26] F.-C. Lei, M. Gao, C. du, Q. L. Jing, and G. L. Long, “Three-pathway electromagnetically induced transparency in coupledcavity optomechanical system," Optics Express, vol. 23, no. 9, pp. 11508-11517, 2015.

[27] M. Stocchi, D. Mencarelli, Y. Pennec, B. Djafari-Rouhani, and L. Pierantoni, "Rigorous simulation of nonlinear optomechanical coupling in micro- and nano-structured resonant cavities," International Journal of Optomechatronics, vol. 12, no. 1, pp. 11-19, 2018.

[28] K. Asadi, J. Yu, and H. Cho, "Nonlinear couplings and energy transfers in micro- and nanomechanical resonators: intermodal coupling, internal resonance and synchronization," Philosophical Transactions of the Royal Society A, vol. 376, no. 2127, p. 20170141, 2018.

[29] S. Hassoul, S. Menouar, H. Benseridi, and J. R. Choi, "Quantum dynamics for a general time-dependent three coupled oscillators," unpublished.

[30] M. J. Kronenburg, "A method for fast diagonalization of a $2 \times 2$ or $3 \times 3$ real symmetric matrix," 2013, https://arxiv.org/abs/ 1306.6291 .

[31] P. B. Denton, S. J. Parke, T. Tao, and X. Zhang, "Eigenvectors from eigenvalues: a survey of a basic identity in linear algebra," 2019, https://arxiv.org/abs/1908.03795.

[32] J. C. Gonzalez-Henao, E. Pugliese, S. Euzzor, R. Meucci, J. A. Roversi, and F. T. Arecchi, "Control of entanglement dynamics in a system of three coupled quantum oscillators," Scientific Reports, vol. 7, no. 1, p. 9957, 2017.

[33] M. V. Putz, Ed., Quantum Nanosystems: Structure, Properties, and Interactions, Apple Academic Press, Florida, 2014.

[34] H. Safavi-Naeini, J. Chan, J. T. Hill, T. P. M. Alegre, A. Krause, and O. Painter, "Observation of quantum motion of a nanomechanical resonator," Physical Review Letters, vol. 108, no. 3, p. 033602, 2012.

[35] C.-Y. Wen, H. Y. Xie, Z. L. Zhang et al., "Fluorescent/magnetic micro/nano-spheres based on quantum dots and/or magnetic nanoparticles: preparation, properties, and their applications in cancer studies," Nanoscale, vol. 8, no. 25, pp. 1240612429, 2016.

[36] A. D. Armour and M. P. Blencowe, "Probing the quantum coherence of a nanomechanical resonator using a superconducting qubit: I. Echo scheme," New Journal of Physics, vol. 10, no. 9, p. 095004, 2008. 